Fisioter Bras 2019;20(3):409-17

https://doi.org/10.33233/fb.v20i3.2801

ARTIGO ORIGINAL

Influência da visão e da dupla tarefa no controle postural de idosas com perdas urinárias Influence of vision and dual task on postural control of elderly women with urinary losses

Joane Severo Ribeiro, M.Sc. ${ }^{*}$ Melissa Medeiros Braz, D.Sc.**, Luiz Fernando Cuozzo Lemos, D.Sc. ${ }^{* *}$, Patrícia Paludette Dorneles, M.Sc. ${ }^{* *}$, Carlos Bolli Mota, D.Sc. ${ }^{* *}$

*Universidade Federal do Rio Grande do Sul (UFRGS), Porto Alegre/RS, **Universidade Federal de Santa Maria (UFSM), Santa Maria/RS, ${ }^{* * * U n i v e r s i d a d e ~ R e g i o n a l ~ I n t e g r a d a ~ d o ~ A l t o ~ U r u g u a i ~} e$ das Missões - URI, Santiago/RS

Recebido em 28 de fevereiro de 2019; aceito em 26 de abril de 2019.

Correspondência: Joane Severo Ribeiro, Rua Carlos Lang, 115/403, 97020130 Santa Maria RS, E-mail: joaneribeiro@gmail.com; Melissa Medeiros Braz: melissabraz@hotmail.com; Patrícia Paludette Dorneles: patriciapaludette@gmail.com; Luiz Fernando Cuozzo Lemos: luizcanoagem@yahoo.com.br; Carlos Bolli Mota:bollimota@gmail.com

\title{
Resumo
}

Introdução: A incontinência urinária (IU) é um processo natural do envelhecimento, afetando 25 a $45 \%$ das mulheres brasileiras. Portadores de IU podem apresentar alterações no controle postural, pois a musculatura do assoalho pélvico é responsável pela estabilização das estruturas da pelve, possuindo íntima relação com o controle postural estático. Objetivo: Comparar o controle postural de mulheres idosas com perdas urinárias e continentes, nas condições de olhos abertos, supressão da visão e teste de dupla tarefa. Métodos: Estudo observacional de caráter transversal com 46 idosas, 26 com IU com 68,31 $\pm 5,79$ anos e 20 continentes com 69,3 $\pm 6,87$ anos. Realizou-se anamnese para identificar aspectos de saúde das idosas, onde incluía autorrelato de perda urinária. Para análise do controle postural utilizou-se uma plataforma de força para a obtenção dos dados referentes ao centro de pressão (COP). A normalidade dos dados foi verificada através do teste de Shapiro-Wilk e teste t de Student. O nível de significância adotado foi de $5 \%$. Resultados: Não foram observadas diferenças significativas em todas as variáveis do COP na situação de olhos abertos, nem nas variáveis $C O P_{a p}$ e $C O P_{m l}$ de olhos fechados, bem como no $\mathrm{COP}_{\mathrm{ap}}, \mathrm{COP}_{\mathrm{ml}}$ e $\mathrm{COP}_{\text {vel }}$ na dupla tarefa. As variáveis $\mathrm{COP}_{\text {vel }}$ e $\mathrm{COP}_{\text {elp }}$ de olhos fechados e COP elp na dupla tarefa, apresentaram diferenças estatisticamente significativas $(\mathrm{p}<0,05)$. Conclusão: As mulheres idosas com relato de IU apresentaram um pior desempenho do controle postural estático no que se refere a velocidade média de oscilação e a área de oscilação, quando comparadas às idosas continentes.

Palavras-chave: equilíbrio postural, dupla tarefa, idosos, incontinência urinária.

\begin{abstract}
Introduction: Urinary incontinence (UI) is a natural process of aging, affecting 25 to $45 \%$ of Brazilian women. Ul patients may present changes in postural control, since the pelvic floor musculature is responsible for the stabilization of the pelvic structures, having an intimate relationship with the static postural control. Objective: To compare the postural control of elderly women with urinary losses and continents, in conditions of open eyes, suppression of vision and double task test. Methods: Observational cross-sectional study with 46 elderly women, 26 with $\mathrm{UI}, 68.31 \pm 5.79$ years and 20 continents, with $69.3 \pm 6.87$ years. Anamnesis was performed to identify aspects of the elderly women's health, including self-report of urinary loss. For analysis of the postural control, a force platform was used to obtain the data related to the pressure center (COP). The normality of the data was verified through the Shapiro-Wilk test and Student's t-test. The level of significance was $5 \%$. Results: There were no significant differences in all COP variables in the open-eyes situation, nor in the $\mathrm{COP}_{a p}$ and $\mathrm{COP}_{\mathrm{ml}}$ variables with eyes closed, as well as in $\mathrm{COP}_{\mathrm{ap}}, \mathrm{COP}_{\mathrm{ml}}$ and $\mathrm{COP}_{\text {vel }}$ in the double task. The COP elp and $\mathrm{COP}_{\text {elp }}$ variables with closed eyes and COP elp in the double task presented statistically significant differences $(p<0.05)$. Conclusion: Older women with UI reported worse performance of static postural control in relation to mean oscillation velocity and oscillation area, when compared to the elderly women.
\end{abstract}


Key-words: postural balance, dual task, elderly, urinary incontinence.

Introdução

Com o aumento da expectativa de vida da população, e sendo os idosos o grupo que mais cresce atualmente, despertou maior interesse com a saúde e a qualidade de vida dos mesmos. Dados do Instituto Brasileiro de Geografia e Estatística [1] mostram que no Brasil já são 23,5 milhões de idosos, e no estado do Rio Grande do Sul esta população equivale a 14,7\% do total. As perspectivas em nível mundial, segundo a Organização Mundial de Saúde [2,3], é que no ano de 2025 a população de idosos será mais de 1,2 bilhão.

O aumento de expectativa de vida das mulheres em relação aos homens nos últimos anos [1] despertou maior preocupação e interesse na saúde e na qualidade de vida desta população. Considerando que as mulheres podem viver mais de um terço de suas vidas após a menopausa, torna-se necessário a compreensão mais aprofundada sobre o processo de envelhecimento feminino e, também, de certa forma, das enfermidades que podem acometer facilmente este grupo.

Dados epidemiológicos apontam que $20 \%$ das mulheres no climatério apresentam perdas urinárias involuntárias, $26 \%$ em fase reprodutiva e esses números sobem para 30 a $40 \%$ na menopausa [4,5]. Segundo a OMS, a incontinência urinária (IU) afeta mais de 200 milhões de pessoas no mundo todo, e é considerado um problema de saúde pública [2,3].

Além disso, com o processo de envelhecimento, a mulher sofre alterações posturais que podem desestruturar sua estrutura pélvica. Com isso, o corpo busca um novo equilíbrio, muitas vezes com danos às funções orgânicas [6]. Assim, os desequilíbrios pélvicos podem levar a um déficit da musculatura perineal e contribuir negativamente, para a continência [7].

Portadores de IU podem apresentar alterações do controle postural $[8,9]$, tendo em vista que a musculatura do assoalho pélvico (MAP) é responsável pela estabilização das estruturas da pelve, e que o processo de envelhecimento desencadeia perdas musculares, assim alterações da MAP podem ter íntima relação com a diminuição da capacidade de manutenção do controle postural estático e dinâmico [10-12]. Dessa forma, é provável que a IU seja associada com uma diminuição da atividade dos MAP, mudanças posturais e consequentemente, alterações de equilíbrio.

Com o aumento da idade, a capacidade de executar determinadas tarefas motoras diminui [13]. O idoso é capaz de manter o equilíbrio postural quando está concentrado na realização de uma atividade específica, mas pode não apresentar o mesmo padrão de execução quando precisa realizar múltiplas tarefas simultaneamente [14,15]. Quando se tem a necessidade da realização concomitante de várias atividades, dividindo a atenção do idoso, isso pode gerar dificuldades no desempenho motor e no controle do equilíbrio [14].

Tendo em vista este contexto, o presente estudo tem por objetivo investigar a influência da visão e da realização do teste de dupla tarefa no controle postural em mulheres com e sem relatos de queixas de perdas urinárias.

Material e métodos

Amostra

Participaram voluntariamente do estudo 46 mulheres idosas, 26 que referiram perdas urinárias diárias e 20 que não referiram perdas urinárias. As idosas responderam a uma ficha de anamnese, em que haviam perguntas referentes ao estado geral de saúde, incluindo o questionamento se apresentavam perdas urinárias, bem como se realizavam tratamento para tal. A amostra não aleatória foi selecionada entre idosas da comunidade e de grupos de conveniência da cidade de Santa Maria/RS, e foram convidadas a participar da pesquisa por meio de cartazes e contato direto nos grupos.

Para o cálculo amostral foi realizado um estudo piloto com 18 sujeitos, utilizando uma das variáveis como parâmetro para a definição do tamanho amostral. O software G*Power 3.1.9.2 foi utilizado para o cálculo da amostra, adotando um tamanho de efeito de 0,8 , intervalo de confiança de $95 \%$ e poder de 80 . Com isso, o tamanho amostral necessário ficou definido com 19 sujeitos por grupo.

Como critério de inclusão os indivíduos deveriam ser do sexo feminino, ter mais de 60 anos de idade, não apresentar patologias que pudessem interferir no resultado do estudo como: 
AVC, doença de Parkinson, distúrbios vestibulares, deficiência visual não corrigida, diabetes mellitus desregulada; fazer uso de dispositivos deambulatórios e apresentar condições cognitivas para responder aos questionamentos de anamnese e questionários.

\section{Questões éticas}

O projeto de pesquisa foi aprovado pelo comitê de ética da UFCSPA com o número de parecer 1710-12 e número de cadastro no CEP 996-12. Todos os participantes que concordaram em participar do estudo assinaram um Termo de Consentimento Livre e Esclarecido (TCLE).

\section{Procedimentos}

Primeiramente foi realizada uma anamnese, a qual foi constituída de uma ficha com dados pessoais, relato de dores e locais, aspectos gerais de saúde, uso de medicamentos, estilo de vida (ingestão de bebidas alcóolicas, cigarro e realização de atividades físicas), relato de perdas urinárias e se estava realizando tratamento em virtude disso. As avaliações foram realizadas numa sala em ambiente silencioso e climatizado, em única visita ao laboratório de biomecânica da Universidade Federal de Santa Maria, LABIOMEC. Após, realizou-se a avaliação antropométrica de massa e estatura do sujeito, por meio de uma balança digital e estadiômetro. Mostrou-se o ambiente em que seriam realizadas as avaliações, e foi feita a explicação de todos os procedimentos previamente e os sujeitos assinaram o TCLE.

Antes da realização da avaliação, os sujeitos ficaram sentados em repouso por cinco minutos. Durante a avaliação, o sujeito de pesquisa foi solicitado a adotar a postura ortostática bipodal sobre a plataforma de força AMTI OR6-6 2000 (Advanced Mechanical Technologies, Inc.) com os pés descalços, onde foi fixada uma folha e desenhada com a posição dos pés a fim de que fosse utilizada a mesma base de apoio para todas as tentativas.

Foram realizadas seis coletas para verificação dos dados de controle postural de cada sujeito. Nas duas primeiras tentativas foi solicitado a cada participante que permanecesse em pé com os olhos abertos (OA) o mais estático possível sobre a plataforma durante 30 segundos, com foco visual fixado em um tripé na altura dos olhos de cada indivíduo a uma distância de 2,70 metros. Os indivíduos repetiram o procedimento em: duas tentativas com os olhos fechados (OF), e mais duas tentativas realizando o teste de dupla tarefa stroop color (SC). Após cada tentativa, pedia-se para o indivíduo sair da plataforma de força, descansar 1 minuto e seguir para a próxima avaliação.

O teste de dupla tarefa foi executado da seguinte forma: foi apresentada uma projeção em uma tela de projeção branca com a escrita de doze nomes de cores, pintadas em cores distintas dos referidos nomes, com tamanho de fonte 60 , distribuídas três por linha, totalizando quatro linhas por slide, após 13 segundos mudava-se o slide para inverter a ordem da distribuição das palavras (Figura 1). A projeção estava à frente dos indivíduos avaliados, a uma distância de 3 metros, numa tela de 2 metros de largura por 1,5 metros de altura, com o projetor conectado a um computador, que gerou e controlou a apresentação de slides. Antes do início das avaliações foi explicada para cada indivíduo a forma de executar o teste de dupla tarefa, devendo ficar o mais parado possível, falar apenas a cor na qual as palavras estavam pintadas e não realizar a leitura da cor. Também foi orientado que a cada mudança do slide se iniciava o texto sempre na primeira linha (não onde se interrompeu o teste/leitura). O início da projeção do teste de dupla tarefa era acionado com um passador de slides, ao mesmo instante da avaliação de controle postural. 


\section{AMARELO LILÁS AZUL}

\section{LARANJA ROSA MARROM}

\section{PRETO AZUL VERRMERSIO}

\section{BRANCO VERDE ROXO}

Figura 1 - Projeção do teste de dupla tarefa stroop color.

Os dados cinéticos foram coletados através da plataforma de força operando a $1000 \mathrm{~Hz}$ e no nível solo. Para filtragem dos dados brutos de força e momento, foi utilizado um filtro passa baixa Butterworth de $4^{\underline{a}}$ ordem com uma frequência de corte de $10 \mathrm{~Hz}$. A partir de tais procedimentos foram calculadas as posições do COP. As variáveis analisadas foram as amplitudes de deslocamento do COP nos eixos anteroposterior ( $\left.C O P_{a p}\right)$ e médio-lateral $\left(C O P_{m l}\right)$, obtidas através diferença entre o valor máximo e mínimo atingido pelo COP em cada direção, a velocidade média do COP ( $\mathrm{COP}_{\text {vel }}$ ), obtida pela divisão do valor de deslocamento total do COP pelo tempo de cada tentativa (30 segundos) e a área de oscilação do COP (COPelp) [16].

A área de oscilação considerada foi a área da elipse $95 \%$, a qual é descrita como a região em que com $95 \%$ de probabilidade contém o centro da oscilação do corpo [17]. Foram utilizadas duas tentativas válidas, escolhidas aleatoriamente, de cada situação e primeiramente foi realizada uma média dos valores das variáveis de cada sujeito de pesquisa, e após uma média geral de todos os indivíduos dentro de cada tentativa.

\section{Análise estatística}

Os dados foram analisados utilizando-se o programa estatístico SPSS 17.0. A normalidade dos dados foi verificada através do teste de Shapiro-Wilk, as características dos indivíduos e as variáveis estudadas foram submetidas à estatística descritiva.

Os dados das variáveis $\mathrm{COP}_{\mathrm{ap}}, \mathrm{COP}_{\mathrm{ml}}, \mathrm{COP}_{\mathrm{vel}}, \mathrm{COP}_{\mathrm{elp}}$, da situação $\mathrm{OA} ; \mathrm{COP}_{\mathrm{ap}}, \mathrm{COP}_{\mathrm{ml}}$, COPvel, COP elp da situação OF; e COPap, COPml, COPvel, COPelp da situação SC apresentaram distribuição normal. Para realizar as comparações entre as situações foi utilizado o teste t de Student para amostra independente.

O nível de significância adotado para todos os testes foi de $5 \%$.

Resultados

O grupo de idosas avaliadas (grupo de idosas incontinentes e idosas continentes), no presente estudo não apresentaram diferenças estatisticamente significativas, no que se refere à idade, estatura, massa e IMC, caracterizando-se por grupos homogêneos entre si. A caracterização das participantes da pesquisa está descrita na Tabela I.

Tabela I - Médias e desvios padrão de idade, dados antropométricos e número de indivíduos em cada grupo.

\begin{tabular}{lllll}
\hline & Idosas IU(n=26) & \multicolumn{3}{l}{ Idosas Continentes $\mathbf{( n = 2 0 )}$} \\
\hline & Média & DP & Média & DP \\
\hline Idade (anos) & 68,31 & 5,79 & 69,3 & 6,87 \\
Estatura (m) & 1,55 & 0,08 & 1,55 & 0,06 \\
Massa $(\mathbf{k g})$ & 67,98 & 11,07 & 65,66 & 11,22 \\
IMC $\left(\mathbf{k g} / \mathbf{m}^{2}\right)$ & 28,39 & 4,21 & 27,40 & 5,56 \\
\hline
\end{tabular}

IU = Incontinência Urinária; DP = Desvio padrão. 
$\mathrm{Na}$ Figura 2, estão representadas as comparações entre os grupos de idosas incontinentes (IU) e continentes (CONT) na situação de olhos abertos (OA). Sendo representado na Figura 2(A) os valores de oscilação ântero-posterior ( $\left.\mathrm{COP}_{\mathrm{ap}}\right)$, na Figura 2(B) oscilação médiolateral $\left(C O P_{\mathrm{ml}}\right)$, na 2(C) os valores da velocidade média de oscilação (COPvel) e na Figura 2(D) os valores da área de oscilação do Centro de Pressão $\left(\mathrm{COP}_{\mathrm{elp}}\right)$. Verificando-se que não houve diferença estatisticamente significativa entre as variáveis do COP na situação de olhos abertos, entre os grupos de idosas analisadas.

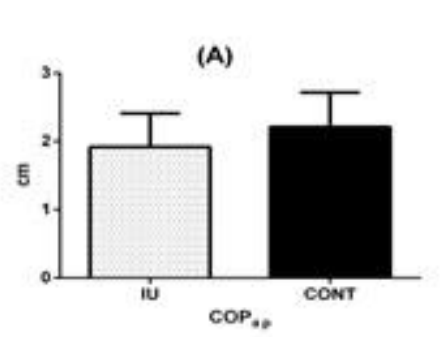

(C)

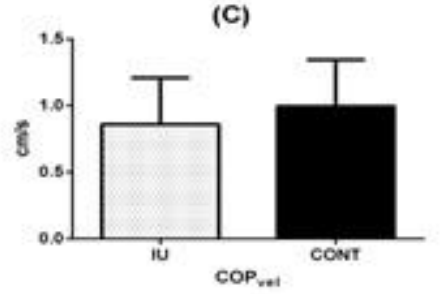

(B) OA

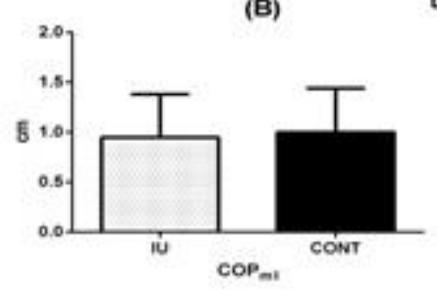

(D)

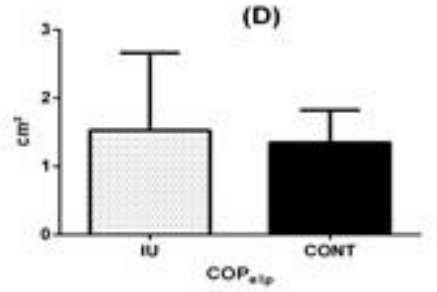

Figura 2 - Comparativo das variáveis do COP, na situação de olhos abertos, entre os grupos de idosas incontinentes e de continentes.

$\mathrm{Na}$ Figura 3, estão representadas as comparações entre os grupos de idosas incontinentes (IU) e continentes (CONT) na situação de olhos fechados (OF). Sendo representado na Figura 3(A) os valores de oscilação ântero-posterior (COPap), na Figura 3(B) oscilação médiolateral $\left(C O P_{\mathrm{ml}}\right)$, na $3(C)$ os valores da velocidade média de oscilação (COPvel) e na Figura 3(D) os valores da área de oscilação do Centro de Pressão (COPelp). Verificando-se que não houve diferença estatisticamente significativa entre as variáveis do $C O P_{\text {ap }}$ e $C O P_{\mathrm{ml}}$. $E$ diferenças estatisticamente significativas entre as variáveis $\operatorname{COP}_{\text {vel }}(p=0,0048)$ e $\operatorname{COP}_{\text {elp }}(p=$ 0,0139) na situação de olhos fechados.

(A)

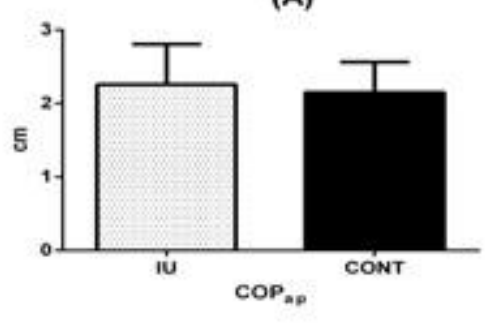

(C)

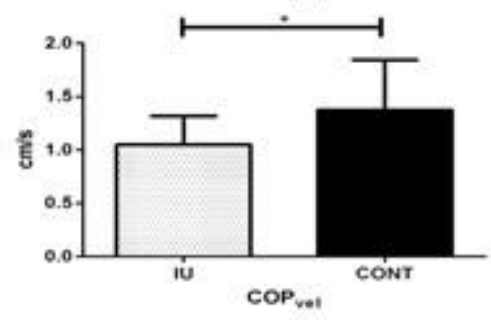

(B)

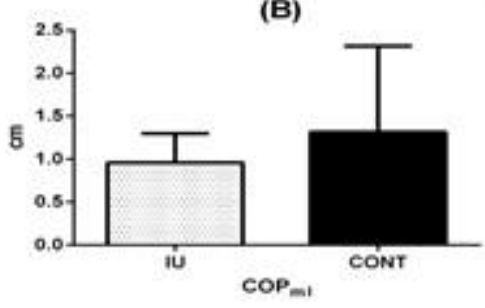

(D)

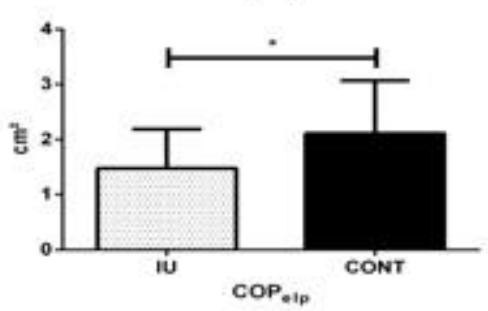

*indica diferença significativa com $p<0,05$

Figura 3 - Comparativo das variáveis do COP, na situação de olhos fechados, entre os grupos de idosas incontinentes e de continentes. 
$\mathrm{Na}$ Figura 4, estão representadas as comparações entre os grupos de idosas incontinentes (IU) e continentes (CONT) na situação de realização de teste de dupla tarefa stroop color (SC). Sendo representado na Figura 4(A) os valores de oscilação ântero-posterior (COPap), na Figura $4(B)$ oscilação médiolateral $\left(C{ } P_{m l}\right)$, na $4(C)$ os valores da velocidade média de oscilação (COPvel) e na Figura 4(D) os valores da área de oscilação do Centro de Pressão $(C O P$ elp). Verificando-se que não houve diferença estatisticamente significativa nas variáveis $\mathrm{COP}_{\mathrm{ap}}, \mathrm{COP}_{\mathrm{ml}}$ e COP vel entre os grupos de idosas analisadas, havendo apenas diferença na variável do $\operatorname{COP}_{\text {elp }}(p=0,0331)$ na situação de realização de teste de dupla tarefa.

(A)

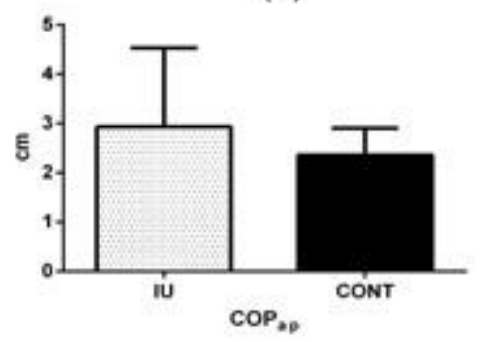

(C)

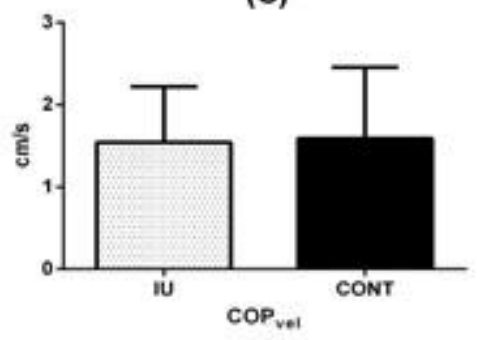

(B)

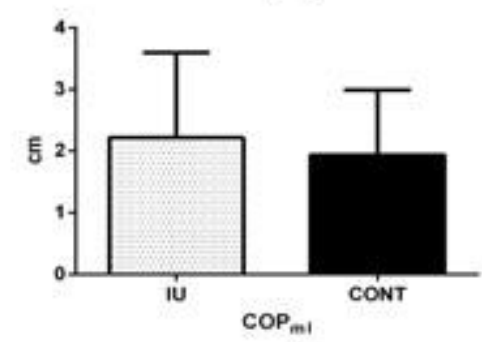

(D)

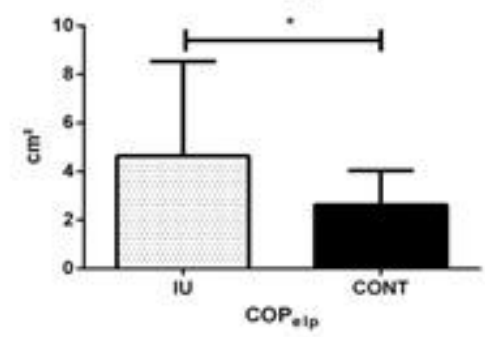

*indica diferença significativa com $p<0,05$.

Figura 4 - Comparativo das variáveis do COP, na situação de realização de teste de dupla tarefa, entre os grupos de idosas incontinentes e de continentes.

\section{Discussão}

O presente estudo teve por objetivo analisar a diferença entre as variáveis do centro de pressão (COP) entre grupos de idosas que tiveram autorrelato de perdas urinárias e de idosas continentes e, desta forma, buscar compreender a relação entre disfunções do assoalho pélvico e a manutenção do controle postural em idosas.

$\mathrm{Na}$ situação de olhos abertos, os achados do presente estudo não apresentaram diferenças de oscilação do centro de pressão, oscilação anteroposterior e médio-lateral, velocidade média de oscilação e a área de oscilação, entre os grupos analisados no presente estudo.

Entretanto, quando se tem a supressão da visão, na situação de olhos fechados, já se pode observar que as idosas com incontinência tiveram maiores valores de velocidade média de oscilação e de área de oscilação, quando comparadas com as idosas que são continentes.

Sabe-se que algumas condições estão relacionadas com a modificação de valores de oscilação corporal. Segundo Masdeu et al. [18], o equilíbrio se deteriora com a idade em decorrência das alterações de diversas funções orgânicas dos idosos como, por exemplo: diminuição da acuidade visual e auditiva, perdas degenerativas no sistema vestibular, redução na flexibilidade, redução da força muscular global e diminuição das informações articulares.

A manutenção do controle postural resulta da interação de diversas características individuais de cada ser humano, como altura, peso corporal, estado emocional, cognição, sistemas sensoriais, força muscular, assim como a tarefa que necessita ser realizada e do ambiente na qual será realizada [19]. Entre essas características que modificam o controle postural destaca-se a incontinência urinária [20].

Estudos, como o de Smith et al. [21], demonstram que há um comprometimento do controle postural em mulheres com IU. O mecanismo está associado ao excesso da ativação da 
musculatura do tronco e do retardo da ativação dos MAP, visto que a musculatura do assoalho pélvico (MAP) faz parte do mecanismo de estabilidade de tronco e sua função é interdependente com outros músculos de outros sistemas [20].

Capson, Nashed e MacLean [22] atribuem aos músculos do assoalho pélvico (MAP), além de suas funções clássicas de manutenção da continência, suporte das vísceras abdominopélvicas e função sexual, as funções de estabilização lombo-pélvica e controle postural. Desta forma, a ativação dos MAP e a coordenação com os músculos do abdome e do tronco desempenham um importante papel no tratamento das mulheres incontinentes.

A ativação dos MAP, em conjunto com os músculos abdominais, pode ser explicada como uma estratégia neural para coordenar estes grupos musculares por inputs descendentes de centros superiores ou mecanismos reflexos espinhais ligados à manutenção da continência quando a pressão intra-abdominal aumenta, pela contração muscular [23].

A coordenação da musculatura dos MAP é fundamental para a continência urinária, a fim de proporcionar um mecanismo ativo de fechamento da uretra e resistir aos aumentos da pressão intra-abdominal [24]. A pré-ativação dos MAP, que antecede qualquer aumento de pressão intra-abdominal, envolve uma atividade neural coordenada que implica o comando central e vias neurais autonômicas [25].

Baseado nisso, alguns estudos avaliaram o controle postural de pessoas com incontinência urinária e continentes [20,26]. Entre esses, o estudo de Smith et al. [21] investigou a diferença no controle postural de mulheres continentes e incontinentes, verificou que as incontinentes apresentam uma maior oscilação do COP quando comparadas às continentes. Tais achados, vão ao encontro dos apresentados do presente estudo, sendo a presença de IU um fator interveniente na manutenção do controle postural das mulheres avaliadas no estudo.

E quando se tem a demanda cognitiva envolvida, as idosas incontinentes apresentam maiores valores de área de oscilação em comparação as continentes. Nesse sentido, alguns estudos demonstram que características como a educação e o QI influenciam na manutenção do controle postural durante a realização de dupla tarefa cognitiva, como o teste stroop color $[17,27]$.

Tarefas cognitivas e de manutenção do controle postural ocorrem a mesmo nível de córtex, o que explicaria a interferência da execução de tarefas concomitantes, havendo prejuízo na execução de uma delas $[27,28]$, como evidenciado no presente estudo, em que o controle postural sofreu interferências significativas durante a realização de uma tarefa cognitiva e visual simultâneas para o grupo incontinente.

A dupla demanda (controle postural e manutenção da continência) nas mulheres com IU aumentam o risco de perdas urinárias, principalmente em tarefas que desafiem o controle postural. Ocorre um atraso na ativação dos MAP durante perturbações posturais, que resultam em um fechamento uretral inadequado [29]. Sapsford e Hodges et al. [23] relatam que, nas mulheres incontinentes, não ocorre a pré-ativação dos MAP antecipatória ao controle postural, quando ocorrem aumentos da pressão intra-abdominal, o que ocorre com as mulheres continentes.

A atenção e a distração parecem desempenhar um papel importante na contração dos MAP durante situações estressantes, significando que a distração mental pode estar envolvida na IU. O estudo de Thubert et al. [30] demonstrou que uma tarefa de distração afetou a capacidade de contração dos MAP, resultando num retardo de ativação deste grupo muscular de 3,98 vezes. Fatores como a presença de dor ou o desempenho de dupla tarefa parecem estar diretamente relacionados à piora da capacidade de pré-ativação dos MAP [25,30].

No que se refere à análise do controle postural de idosas incontinentes, é importante levar em consideração que o desempenho da dupla tarefa pode constituir risco de quedas para o grupo pesquisado, o que seria de fundamental importância para a clínica, de modo a auxiliar nos serviços de atendimentos aos idosos, como educação em saúde e nos processos de reabilitação.

Tais distinções seriam de fundamental importância para a clínica, de modo a auxiliar nos serviços de atendimentos aos idosos, como educação em saúde e nos processos de reabilitação. 
Com base nos resultados do presente estudo, pode-se concluir que idosas com incontinência urinária apresentaram maiores oscilações do centro de pressão, quando comparadas com idosas continentes. Evidenciando que a musculatura do assoalho pélvico se relaciona com o controle postural, pois é responsável pela estabilização das estruturas da pelve e possui íntima relação com o equilíbrio estático.

Os autores gostariam de agradecer ao LABIOMEC e ao CNPq.

1. Instituto Brasileiro de Geografia e Estatística (IBGE). Sinopse do Censo Demográfico 2010. 30 de junho de 2012. Rio de Janeiro: IBGE; 2012.

2. Organização Mundial de Saúde (OMS). Growing older. Staying well. Ageing and physical activity in everyday life. Preparado por Heikkinen RL. Genebra: Organização Mundial da Saúde; 1998.

3. Organização Mundial de Saúde (OMS). Active ageing: A policy framework. Second United Nations World Assembly on Ageing. Madrid, Spain, abr. 2002. [citado $2016 \mathrm{Jul}$ 12]. Disponível em: http://www.who.int/hpr/ageing/ActiveAgeingP olicyFrame.pdf.

4. Paschoal SMS. Qualidade de vida do idoso: elaboração de um instrumento que privilegia sua opinião [Dissertação]. São Paulo: USP; 2000.

5. Reis RB, Cologna AJ, Martins ACP, Paschoalin EL, Tucci JRS, Suaid HJ. Incontinência urinária no idoso. Acta Cir Bras 2003;18(5):47-51. https://doi.org/10.1590/S010286502003001200018

6. Wallach S, Ostergard D. Anatomia pélvica feminina. In: D’Ancona CAL, Netto Júnior NR, eds. Aplicações clínicas da urodinâmica. 3 ed. São Paulo: Atheneu; 2001. p.12638.

7. Perry JD, Hullet TL. Urinary incontinence and pelvic muscle rehabilitation index. Northeastern Gerontological Society. New Brunswick/NJ; 1998. p.20.

8. Horak FB, Macpherson JM. Postural orientation and equilibrium. In Rowell LB, Sherpherd JT, eds. Handbook of physiology: a critical, comprehensive presentation of physiological knowledge and concepts. Oxford American Physiological Society; 1996. p.255-92.

9. Maki BE, Mcilroy WE, Fernie GR. Change-in-support reactions for balance recovery. IEEE Eng Med Biol Mag 2006;22(2):20-6.

10. Araújo THP, Francisco LPT, Leite RF, lunes DH. Posicionamento da pelve e lordose lombar em mulheres com incontinência urinária de esforço. Fisioter Pesqui 2010;17(2):130-5.

11. Fozzatti MCM, Palma P, Herrmann V, Dambros M. Impacto da Reeducação Postural Global no tratamento da incontinência urinária de esforço feminina. Rev Ass Med Bras 2008;54(1):17-22. https://doi.org/10.1590/S0104-42302008000100015

12. Etienne MA, Watman MC. Disfunções sexuais femininas: a fisioterapia como recurso terapêutico. São Paulo: LMP; 2006.

13. Kenny GP, Yardley JE, Martineau L, Jay O. Physical work capacity in older adults: Implications for the aging worker. Am J Ind Med 2008;51:610-25. https://doi.org/10.1002/ajim.20600

14. Jamet M, Deviterne D, Gauchard GC, Vançon G, Perrin PP. Age-related part taken by attentional cognitive processes in standing postural control in a dual-task context. Gait Posture 2006;25(2):179-184. https://doi.org/10.1016/i.gaitpost.2006.03.006

15. Voelcker-Rehage C, Alberts JL. Effect of motor practice on dual-task performance in older adults. J Gerontol B Psychol Sci Soc Sci 2007;62(3):141-8. https://doi.org/10.1093/geronb/62.3.p141

16. Duarte M, Freitas SMSF. Revisão sobre posturografia baseada em plataforma de força para avaliação do equilíbrio. Rev Bras Fisioter 2010;14:183-192.

17. Rocchi MBL, Sisti D, Ditroilo M, Calavalle A, Panebianco R. The misuse of the confidence ellipse in evaluating statokinesigram. Ital J Sport Sci 2005;12:169-72. 
18. Masdeu JC, Sudarsky L, Wolfson L. Gait disorders of aging. Philadelphia LippincottRaven; 1997.

19. Soares AV. A contribuição visual para o controle postural. Rev Neurocienc 2010;18(3):370-9.

20. Sapsford RR, Richardson CA, Maher CF, Hodges PW. Pelvic floor muscle activity in different sitting postures in continent and incontinent women. Arch Phys Med Rehabil 2008;89(9):1741-7. https://doi.org/10.1016/j.apmr.2008.01.029

21. Smith MD, Michel W, Coppieters MW, Hodge PW. Is balance different in women with and without stress urinary incontinence? Neurourol Urodyn 2008;27(1):71-8. https://doi.org/10.1002/nau.20476

22. Capson AC, Nashed J, Mclean L. The role of lumbopelvic posture in pelvic floor muscle activation in continent women. J Electromyogr Kinesiol 2011;21(1):166-77. https://doi.org/10.1016/i.jelekin.2010.07.017

23. Sapsford RR, Hodges PW. The effect of abdominal and pelvic floor muscle activation on urine flow in women. Int Urogynecol J 2012;23:1225-30. https://doi.org/ 10.1007/s00192-011-1654-2

24. Verelst M, Leivseth G. Are fatigue and disturbances in pre-programmed activity of pelvic floor muscles associated with female stress urinary incontinence? Neurourol Urodyn 2004;23:143-7. https://doi.org/ 10.1002/nau.20004

25. Thubert T, Deffieux X, Jousse M, Guinet-Lacoste A, Ismael SS, Amarenco G. Influence of a distraction task on pelvic floor muscle contraction. Neurourol Urodyn 2015;34(2):139-43. https://doi.org/10.1002/nau.22524

26. Ueda LS, Carpes FP. Relação entre sensibilidade plantar e controle postural em jovens e idosos. Rev Bras Cineantropom Desempenho Hum 2013;15(2):215-224. http://doi.org/10.5007/1980-0037.2013v15n2p215

27. Barbosa JMM, et al. Efeito da realização simultânea de tarefas cognitivas e motoras no desempenho funcional de idosos da comunidade. Fisioter Pesqui 2008;15(4):374-9. https://doi.org/10.1590/S1809-29502008000400010

28. Hauer K, Marburger C, Oster P. Motor performance deteriorates with simultaneously performed cognitive tasks in geriatric patients. Arch Phys Med Rehabil 2002;83(2):21723.

29. Smith LK, Weiss El, Lehmkuhl, ID. Cinesiologia clínica de Brunnstrom. 5ª ed. São Paulo: Manole; 1997. p.538.

30. Thubert T, Villot A, Billecocq S, Auclair L, Amarenco G, Deffieux X. Influence of a distraction task on the involuntary reflex contraction of the pelvic floor muscles following cough. Neurourol Urodyn 2015;36(1):160- 5. https://doi.org/10.1002/nau.22903 